\begin{tabular}{l} 
O P E R A T I O N S R E S E A R C H A N D D E C I S I O N S \\
\hline No. 4
\end{tabular}

DOI: $10.37190 /$ ord200402

\title{
INTUITIONISTIC FUZZY SETS IN ASSESSING THE MARGINAL VALUE OF THE ELEMENTS OF A MULTIGRAPH
}

\author{
BARBARA GŁADYSZ ${ }^{1 *}$, JACEK MERCIK ${ }^{2}$ \\ ${ }^{1}$ Wrocław University of Science and Technology, Łukasiewicza 5, 50-371 Wrocław, Poland \\ ${ }^{2}$ WSB University in Wroclaw, Fabryczna 29-31, 53-609 Wrocław, Poland
}

\begin{abstract}
The paper presents a method of assessing the value of individual elements of the multigraph, i.e., the value of its vertices and arcs, considering the fuzziness of its parameters. The need to take into account the specificity of the existing types of vertices (including logical functions specified on them) and the possibility of multiple relationships between two neighbouring vertices make it necessary to use a multigraph. The assumed basis for the evaluation of the individual elements of the multigraph was their marginal value, which is the so-called contribution of a given element to the entire multigraph, assuming that the given element affects not only the adjacent elements directly related to it but in a way, perhaps indirect, every other element of the multigraph.
\end{abstract}

Keywords: communication system, multigraph, intuitionistic set, marginal value

\section{Introduction}

Modelling the situation in which there are sets of elements and relationships connecting these elements is common and does not require justification. The reason for such modelling is most often the need to understand both the phenomenon itself and the rules that govern it. Therefore, a sort of optimisation is expected to take place, e.g., profit maximisation or cost minimisation, to mention only the most common optimisations. Moreover, the relationships themselves can be different. They can be physical (e.g., flow of money or goods), logical (e.g., order of technological operations) or mixed (i.e., both physical and logical simultaneously, or in parallel). It seems that a multigraph, i.e., a graph with the possibility of multiple parallel arcs existing

*Corresponding author, email address: barbara.gladysz@pwr.edu.pl Received 9 April 2020, accepted 4 December 2020 
between two neighbouring vertices is the best tool for building a model to describe such situations.

Describing real phenomena with the help of a multigraph, one should imagine the possibility of performing simulation analyses assuming the possibility of changing both the vertices and their connections. This, in turn, creates the need to find the role that the given elements (graph vertices) play in a given graph (multigraph), as well as the connections between them (graph arcs). Thus, defining the role of graph elements should not only be descriptive, but also quantitative, and its simple consequence should be a measure of the importance of individual elements of the graph (multigraph), i.e., the importance of vertices and arcs, taking into account their weights. Such a measure is both absolute, referring directly to the elements of the multigraph (vertices and arcs), and relative, resulting from the overall nature of the relationships between them. In other words, e.g., the capacity of a connection between two vertices has absolute value, resulting from the very nature of the connection, as well as relative value by affecting other connections in each graph.

In the proposed estimation of the importance of graph elements, we propose a methodology associated with cooperative games and we propose to use the modified Shapley value [11] as a measure of importance, in particular the marginal value of the coalition element. The fact that the vertices in a graph are connected through relationships means that we can build specific subsets of interrelated vertices, which, in a game theory, are called coalitions. Since not all the coalitions have the same probability of realising the Shapley value, we modify these probabilities following the estimates of relevant probabilities resulting from the dependencies between individual elements of a given coalition. The beginning of this approach can be found in the work of Owen [10] where the concept of pre-coalition, i.e., the initial coalition resulting from the structure of dependence (in our case the structure of relations between individual vertices of the graph [9]) was used. There is extensive literature on various modifications of the Shapley value, a discussion of which can be found, e.g., in [12].

As already mentioned, both the vertices of the multigraph and their arcs describe selected parameters. For a long time, it has become clear in applications that these parameters are not usually determined values. Since these parameters refer to real processes, often with poor repeatability, which excludes the use of statistical methods, it has become necessary to apply methods of estimation alternative to stochastic descriptions. We believe that the approach resulting from fuzzy set theory [15] can serve as a convenient modelling tool. Gładysz and Mercik [4] in their paper present a fuzzy approach to assessing the value of graph elements. Forlicz et al. [2] present the concept of the Shapley value for multigraphs, while Gładysz et al. [4, 5] present a fuzzy approach to assessing communication graph parameters. A discussion of the applications of such approaches include, for example, Gladysz et al. [3]. In the presented paper, we use another fuzziness modification to evaluate multigraph parameters, i.e., intuitionistic fuzzy sets [1]. 


\section{Preliminaries}

\subsection{Intuitionistic fuzzy sets}

Intuitionistic fuzzy sets were proposed by Attanassov [1].

Definition 1. An intuitionistic fuzzy set is a fuzzy set $A$ in space $U$ so that

$$
A=\left\{\left(x, \mu_{A}(x), v_{A}(x)\right) ; x \in U\right\}
$$

where $\mu_{A}: U \rightarrow[0,1], v_{A}: U \rightarrow[0,1]$ in which $\mu_{A}(x)$ specifies the possibility that $x$ belongs to set $A$, whereas $v_{A}(x)$ specifies the possibility that $x$ does not belong to set $A$. Moreover, $\mu_{A}(x)+v_{A}(x) \leq 1$.

The pair $\left\langle\mu_{A}(x), v_{A}(x)\right\rangle$ is called the intuitionistic fuzzy value of element $x$ in set $A$. The value $\pi_{A}(x)=1-\mu_{A}(x)-v_{A}(x)$ is called the degree of non-determinacy or uncertainty of the element $x \in U$ of the intuitionistic fuzzy set $A$. This value is also called the hesitation value of $x$.

Definition $2[14,13]$. Let $E_{j}=\left(\mu_{E_{j}}, v_{E_{j}}\right), j=1,2, \ldots, n$ be a collection of intuitionistic fuzzy values, and an intuitionistic fuzzy weighted averaging operator of dimension $n$ is shown as

$$
I F W A_{w}\left(E_{1}, E_{2}, \ldots, E_{n}\right)=\left(1-\prod_{j=1}^{n}\left(1-\mu_{E_{j}}\right)^{w_{j}}, \prod_{j=1}^{n} v_{E_{j}}^{w_{j}}\right)
$$

\subsection{Elements of a multigraph}

Definition 3. Let $G\{N, L, k\}$ be a connected graph ${ }^{1}$ in which $N$ is a set of vertices $N=1,2, \ldots, n$ and $L$ is a set of arcs between these vertices, $L=\left\{\left(i_{k}, j_{k}\right), i, j=1,2, \ldots, n\right.$, $k=1\}$. If $i_{k}=j_{k}$, then there is a loop around the vertex end. A multigraph is a graph in which there can be more than one relationship (connection) between two adjacent vertices, i.e., it is a graph for which $k \geq 1$. graph.

${ }^{1} \mathrm{By}$ connected graph we mean that there is a symmetrical path connecting any two vertices of the 
Both to vertices $N$ and $\operatorname{arcs} L$, we assign respective non-negative weights $w_{i} \in \mathcal{R}$, and $w_{i_{k}, j_{k}}$, respectively ${ }^{2}$, while $\sum_{i \in N} w_{i}+\sum_{i_{k}, i_{j} \in L} w_{i_{k}, j_{k}}=1$. Depending on the context, these weights can be given different interpretations. In addition, we assign the graph $G$ a collection of intuitionistic fuzzy values defining the specificity of the cooperation of its elements:

$$
E=\left\{\left(e, \mu_{G}(e), v_{G}(e)\right) ; e \in N \cup L\right\}
$$

where: $\mu_{G}(e)$ specifies the possibility that element $e$ is an element of graph $G$, while $v_{G}(e)$ specifies the possibility that $e$ is not an element of graph $G$.

These sets can be interpreted depending on the decision problem described by the graph. For example, $\mu_{G}(e)$ can mean the possibility that a given graph element $e \in N \cup L$ will cooperate, while $v_{G}(e)$ the possibility of lack of cooperation of an element $e$.

For example, if a graph describes dependencies in the ownership structure of many companies characterised by cross-shareholding, likely multiple relationships, and places their representatives in the management bodies as part of the execution of their ownership rights, then the impact of these representatives on the market value of a given company can only be judged indirectly; however, it is uncertain whether they can be classified as factors increasing or decreasing the market value of the company. An example of such considerations can be found, e.g., in the works of Mercik, Łobos [7] or Mercik, Stach [8].

Definition 4. A connected multigraph will be called a graph in which for each pair of vertices $i, j \in N$ there is a path from $i$ to $j$, and a path from $j$ to $i$.

Definition 5. A connection or vertex from a connected multigraph $G$ is called pivotal in a graph $G$ if its removal from the multigraph causes that a graph $G$ is no longer a connected graph.

\section{Marginal value of an element of the multigraph}

Similarly to the definition of the Shapley value, we will now show the marginal value for a given multigraph element. In general, by the marginal value of a given multigraph element, we mean the difference between the value of the graph containing the

${ }^{2}$ For example, Mercik [6] defines the vertex weight as the weighted average weight of the connections linked to this vertex. 
given element and the value of the graph without that element. It is obvious that, as a consequence, we can expect that (again, as in the Shapley value) it will be possible to determine the value of a given element in the whole multigraph.

Let $M=\operatorname{card}\{G\}$, i.e., $M$ is the number of vertices and connections present in a graph (multigraph) $G$. Note that $M \geq 3$ regardless of which graph we are dealing with.

Definition 6. The intuitionistic fuzzy value $I F W A v_{G}$ of a connected multigraph $G=\{N, L, k\}$ is called

$$
I F W A v_{G}=I F W A_{w}\left(e_{1}, e_{2}, \ldots, e_{M}\right)=1-\prod_{j=1}^{M}\left(1-\mu_{e_{j}}\right)^{w_{j}^{\text {norm }}}, \prod_{j=1}^{M} v_{e_{j}}^{w_{j}^{\text {norm }}}
$$

where: $w_{j}^{\text {norm }}=\frac{w_{j}}{\sum_{e_{j} \in N \cup L} w_{j}}, e_{j} \in N \cup L, j=1, \ldots, M$.

Definition 7. The value $v_{G}$ of the multigraph $G=\{N, L, k\}$ is

- for a connected graph

$$
v_{G}=1-\pi\left(I F W A v_{G}\right)=1-\left(\prod_{j=1}^{M}\left(1-\mu_{e_{j}}\right)^{w_{j}^{\text {norm }}}\right)+\prod_{j=1}^{M} v_{e_{j}}^{w_{j}^{\text {norm }}}
$$

- for a disconnected graph $v_{G}=0$.

This value is defined as ( 1 - non-determinacy) of an intuitionistic fuzzy weighted averaging operator, therefore it informs about the total weighted expected information we have about the sum of the compliant and non-compliant functioning of the multigraph's structure $G$.

Definition 8. Let $e$ be any element of the graph $G=\{N, L, k\}$, i.e., the vertex or connection appearing in this graph. The marginal value of element $e$ is

$$
v_{G}(e)=v_{G}-v_{G\{\{e\}}
$$

Note that if a given element $e \in G$ is a pivotal element, its marginal value is equal to the value $v_{G}$ of a graph $G$. It results directly from the fact that the removal of a pivotal element in a multigraph turns it into a disconnected graph (multigraph), for which the value as a whole is 0 (however, we can talk about separate values of newly created separate parts, if they are connected). 
The marginal value of an element is also a measure of the importance of the element in a given graph structure. On the one hand, it takes into account the weight of the element as such and, on the other hand, its role in the graph structure. The positive (negative) marginal value $v_{G}(e)$ of the multigraph element $e$ tells us how much the average uncertainty about the possibility of cooperation/non-cooperation of the multigraph elements after deleting the element $e$ increases (decreases). Zero marginal value of the element $e$ means that it is a neutral element, i.e., that the multigraph $G$ and the graph $G \backslash e$ have the same hesitation as to the average possibility of cooperation/non-cooperation of the elements of these graphs.

\section{Example}

Let us consider the example of a multigraph in Fig. 1. There are 3 vertices and 5 relationships represented by arcs in this graph. Note that one of these relationships is a loop (b5) and one is an asymmetrical directed relationship (b3). Other relationships are not directed. Besides, relationships $b 1$ and $b 2$ are multiple (in this case double) relationships between vertices $A 1$ and $A 2$.

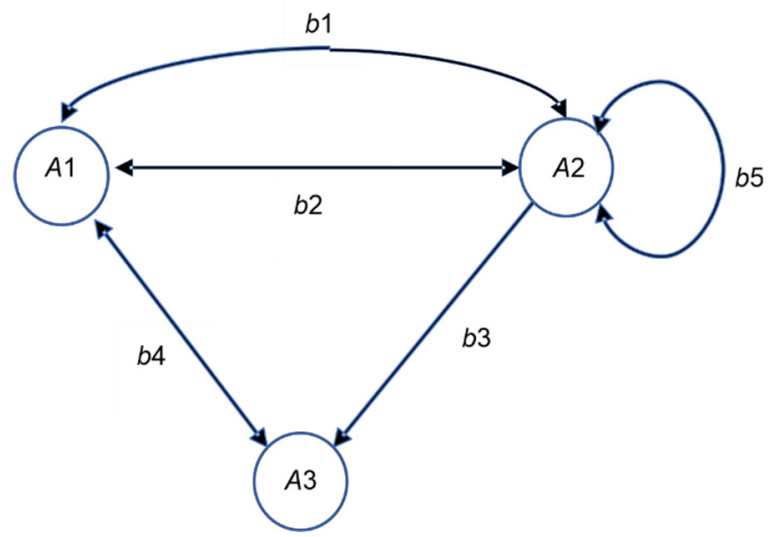

Fig. 1. A multigraph $G$ of three vertices $(A 1, A 2, A 3)$ and five connections, of which $b 1$ and $b 2$ are parallel connections, $b 5$ connection creates a loop, and $b 3$ connection is directed; source: [2]

Let us assign intuitionistic fuzzy values and weights to individual elements of the multigraph $G$ (Table 1). Intuitionistic fuzzy values define the possibility that a given multigraph element will behave as expected. For example, vertex $A 1$ has a weight value of 5. At the same time, we estimate that the possibility that the vertex $A 1$ described by a weight equal to 5 will reach this value is 0.7 and that the possibility that it will not reach it is 0.2 . If the weight $w_{e}$ were to describe, e.g., the expected value of transmitted information in the vertex, then 0.7 means the possibility that the value of the transmitted 
information (the effect of cooperation) will be equal to 5 . In this case, the value of 0.2 signifies the possibility that the value of the transmitted information will be less than 5 , or it will not occur at all, which means an emergency operation of a given element, contrary to the assumptions.

Table 1. Intuitionistic fuzzy values, hesitations, and weights of the elements of the multigraph from Fig. 1

\begin{tabular}{|c|c|c|c|c|c|}
\hline No. & $\begin{array}{c}\text { Element } e \\
\text { of graph } G\end{array}$ & $\begin{array}{c}\text { Intuitionistic fuzzy } \\
\text { value }\left(\mu_{e}, v_{e}\right)\end{array}$ & $\begin{array}{c}\text { Hesitation } \\
\pi_{e}\end{array}$ & $\begin{array}{c}\text { Weight } \\
w_{e}\end{array}$ & $\begin{array}{c}\text { Normalised } \\
\text { weight } w_{e}^{\text {norm }}\end{array}$ \\
\hline 1 & $A 1$ & $(0.7,0.2)$ & 0.10 & 5 & 0.10 \\
\hline 2 & $A 2$ & $(0.5,0.3)$ & 0.20 & 7 & 0.14 \\
\hline 3 & $\mathrm{~A} 3$ & $(0.6,0.2)$ & 0.20 & 1 & 0.02 \\
\hline 4 & $b 1$ & $(0.75,0.2)$ & 0.05 & 8 & 0.16 \\
\hline 5 & $b 2$ & $(0.5,0.2)$ & 0.30 & 9 & 0.18 \\
\hline 6 & $b 3$ & $(0.6,0.2)$ & 0.20 & 4 & 0.08 \\
\hline 7 & $b 4$ & $(0.75,0.1)$ & 0.15 & 8 & 0.16 \\
\hline 8 & $b 5$ & $(0.8,0.1)$ & 0.10 & 8 & 0.16 \\
\hline
\end{tabular}

Table 1 also lists hesitation for individual graph elements, otherwise known as the degree of non-determinacy or uncertainty of the element $e \in N \cup L$ of the intuitionistic fuzzy set $A$. In the analysed example, this value determines the lack of knowledge about the operation of a given graph element (uncertainty about the behaviour of a given element), i.e., the knowledge of whether it will behave following or not expectations. Again, if the weight of the vertex $A 1$ was 5 and described the expected value of the transmitted information, the hesitation value of 0.1 assigned to it means that the uncertainty as to the behaviour of this element (consistent with expectations or not) is assessed as 0.1 on a scale of 0 to 1 . As can be seen, there is a value describing the cooperation of a given graph element with other elements.

Continuing the analysis of the results obtained for the analysed example (Table 1), we find that the greatest possibility of cooperation (0.8) is characterised by the arc (loop) $b 5$ which is probably not surprising since cooperation with oneself is a fairly obvious feature. The smallest assessment of cooperation (0.5) is that of each node $A 2$ and arc $b 2$ separately, which is not an obvious result, and it indicates the sensitivity of these elements in the set of all elements of the analysed multigraph. We should also note that at the same time $b 2$ arc has the highest hesitation value (0.3), i.e., it is characterised by the greatest uncertainty as to its behaviour, which coincides with the previous conclusion, regarding the values relating to the cooperation of $b 2$ element with the rest of the elements of the analysed multigraph.

According to equation (1), the intuitionistic fuzzy value of multigraph $G$ shown in Fig. 1 is $(0.68,0.17)$ and hesitation is equal to 0.15 . In this case, the hesitation value indicates a relatively low "inability" to meet the expectations of the entire analysed multigraph. 
We also note that e.g., the vertex $A 1$ is a pivotal vertex because its removal causes the division of the graph $G$ into two consistent subgraphs: $A 3$ and $A 2$ (there is a path connecting vertex $A 2$ with vertex $A 3$ but there is no symmetrical path, i.e., connecting vertex $A 3$ with vertex $A 2$ ). Hence its relative importance in the analysed multigraph. Based on the data referring to the analysed multigraph (Table 1), intuitionistic fuzzy values and marginal values of individual graph elements were calculated (Table 2).

Table 2. Intuitionistic fuzzy values of multigraphs and marginal values of the elements of the analysed multigraph

\begin{tabular}{|c|c|c|c|c|c|}
\hline No. & $\begin{array}{c}\text { Element } e \\
\text { of multigraph } G\end{array}$ & $\begin{array}{c}\text { Intuitionistic fuzzy } \\
\text { value of multigraph } G \\
\text { without element } e\end{array}$ & $\begin{array}{c}\text { Hesitation } \\
\text { of multigraph } G \\
\text { without element } e\end{array}$ & $\begin{array}{c}\text { Multigraph value } \\
\text { without element } e\end{array}$ & $\begin{array}{c}\text { Marginal values } \\
\text { of element } e\end{array}$ \\
\hline 1 & $A 1$ & disconnected graph & - & 0 & 0.85 \\
\hline 2 & $A 2$ & disconnected graph & - & 0 & 0.85 \\
\hline 3 & $\mathrm{~A} 3$ & disconnected graph & - & 0 & 0.85 \\
\hline 4 & $b 1$ & $(0.67,0.16)$ & 0.17 & 0.83 & 0.02 \\
\hline 5 & $b 2$ & $(0.71,0.16)$ & 0.13 & 0.87 & -0.02 \\
\hline 6 & $b 3$ & $(0.68,0.17)$ & 0.15 & 0.85 & 0 \\
\hline 7 & $b 4$ & disconnected graph & - & 0 & 0.85 \\
\hline 8 & $b 5$ & $(0.65,0.19)$ & 0.16 & 0.84 & 0.01 \\
\hline \multicolumn{2}{|r|}{ Multigraph $G$} & $(0.68,0.17)$ & 0.15 & 0.85 & \\
\hline
\end{tabular}

Analysing the results presented in Table 2, we note that the highest marginal value (0.85) of multigraph $G$ is that of vertices $A 1, A 2, A 3$, and arc $b 5$. These are also pivotal elements because after removing them from multigraph $G$ we receive disconnected graphs.

Arc $b 1$ of multigraph $G$ has a positive marginal value of 0.02 . This means that after its removal from multigraph $G$, we get graph $G \backslash b 1$ whose hesitation is greater than the hesitation of a complete multigraph. Similarly, arc $b 5$ has a positive marginal value of 0.01. In other words, after removing these arcs from the graph, there decreases the amount of information we have about the possibility of cooperation (and non-cooperation) of the elements of communication systems $G \backslash b 1$ and $G \backslash b 5$ decrease by 0.02 and 0.01 , respectively.

Arc $b 3$, however, has a zero marginal value. Therefore, after its removal from the multigraph, the hesitation of the multigraph does not change. It is a directed arc (from vertex $A 2$ to vertex $A 1$ ) with a low weight of 4 . Removing $b 2$ arc reduces the hesitation of the $G \backslash b 2$ graph by 0.02 . The marginal value of this element is 0.02 . Thus, our knowledge about the possibility of cooperation (and non-cooperation) of graph $G \backslash b 2$ elements is greater by 0.02 than the information we have about the cooperation (and non-cooperation) of multigraph $G$ elements. 


\section{Conclusions and plans}

The presented concept of the marginal value of the multigraph elements allows the assessment of the importance of individual elements of the multigraph in the conditions of uncertainty of the behaviour of its elements. The observed uncertainty is modelled with the use of intuitionistic fuzzy sets, which seems to be the best tool in a situation where the data describing individual elements of the system are not statistical though undetermined.

The use of an approach analogous to the Shapley value in cooperative games ensures that the obtained solution is best and meets expectations related to such a measure of uncertainty of multigraph elements. At the same time, the entire calculation process can be algorithmised, and thus used in the description of existing systems.

Separate attention should be focused on hesitation values, which can be a singlenumber characteristic of complex systems that can be presented in the form of a multigraph with intuitive parameters. Soon, we intend to deal with the axiomatisation of such a measure.

\section{References}

[1] AtTanassov K.T., Intuitionistic Fuzzy Sets: Theory and Applications, Physica-Verlag, Springer, Heidelberg 1999.

[2] Forlicz S., MerciK J., Stach I., RamSEy D., The Shapley value for multigraphs [In:] N.T. Nguyen, E. Pimenidis, Z. Khan, B. Trawiński (Eds.), Computational Collective Intelligence, ICCCI, 2018, Lecture Notes in Computer Science, Vol. 11056, Springer, Cham, 213-221.

[3] Gladysz B., Mercik J., Ramsey D., A Fuzzy Approach to Some Shapley Value Problems in Group Decision Making, [In:] E. Algaba, V. Fragnelli, J. Sanchez-Soriano (Eds.), Handbook of the Shapley Value, CRC Press, Taylor and Francis Group, 2020, 483-514.

[4] GŁadysz B., MerCIK J., The Shapley value in fuzzy simple cooperative games, [In:] N.T. Nguyen, D. Hoang, T.P. Hong, H. Pham, B. Trawiński (Eds.), Intelligent Information and Database Systems, ACIIDS 2018, Lecture Notes in Computer Science, Vol. 10751, Springer, Cham, 410-418.

[5] GŁadysz B., MerciK J., Stach I., Fuzzy Shapley value-based solution for communication network, [In:] N. Nguyen, R. Chbeir, E. Exposito, P. Aniorté, B. Trawiński (Eds.), Computational Collective Intelligence, ICCCI, 2019, Lecture Notes in Computer Science, Vol. 11683, Springer, Cham, 535-544.

[6] MERCIK J., A power-graph analysis of non-fast information transmission, [In:] N.T. Nguyen, S. Toyo, L.M. Nguyen, B. Trawiński (Eds.), Intelligent Information and Database Systems, Part I, ACIIDS, 2017, Lecture Notes in Computer Science, Vol. 10191, 89-99, DOI: 10.1007/978-3-319-54472-4_9.

[7] MERCIK J., ŁOBOS K., Index of implicit power as a measure of reciprocal ownership, Trans. Comp. Coll. Int., 2016, 23, 128-140.

[8] MERCIK J., STACH I., On measurement of control in corporate structures, [In:] N. Nguyen, R. Kowalczyk, J. Mercik, A. Motylska-Kuźma (Eds.), Transactions on Computational Collective Intelligence, TCCI, 2018, Lecture Notes in Computer Science, Vol. 11290, Springer, Berlin, 64-79.

[9] MYerson R., Graphs and cooperation in games, Math. Oper. Res., 1997, 2, 225-229. 
[10] Owen G., Values of games with a priori unions, [In:] R. Henn, O. Moeschlin (Eds.), Mathematical Economics and Game Theory, Springer-Verlag, Berlin 1977, 76-88.

[11] Shapley L.S., A value for n-person games, [In:] H. Kuhn, A.W. Tucker (Eds.), Contributions to the Theory of Games II, Princeton University Press, Ann. Math. Studies, 1953, 28, 307-317.

[12] Stach I., Sub-coalitional approach to values, Springer Trans. Comp. Coll. Int., 2017, 10480, $75-87$.

[13] WANG F., ZENG S.L., ZHANG C.H., A method based on intuitionistic fuzzy dependent aggregation operators for suppliers selection, Math. Probl. Eng., 2013, Article ID 481202.

[14] Xu Z.S., Intuitionistic fuzzy aggregation operator, IEEE Trans. Fuzzy Syst., 2007, 15 (6), 1179-1187.

[15] ZADEH L.A., Fuzzy sets, Inf. Control, 1965, 8, 338-353. 